\title{
Preface to CMLSA 2008
}

\author{
Yi-Ping Phoebe Chen ${ }^{1}$, Sven Hartmann ${ }^{2}$, and Markus Kirchberg ${ }^{3}$ \\ ${ }^{1}$ Deakin University, Australia \\ ${ }^{2}$ Clausthal University of Technology, Germany \\ ${ }^{3}$ Institute for Infocomm Research, A*STAR, Singapore
}

Life Sciences applications typically involve large volumes of data of various kinds and a multiplicity of software tools for managing, analysing and interpreting them. There are many challenging problems in the processing of life sciences data that require effective support by novel theories, methods and technologies. Conceptual modelling is the key for developing high-performance information systems that put these theories, methods and technologies into practice. The fast growing interest in life sciences applications calls for special attention on resource integration and collaborative efforts in information systems development.

This volume contains the papers presented at the Second International Workshop on Conceptual Modelling for Life Sciences Applications (CMLSA 2008) which was held in Barcelona, Spain from October 20 to 23, 2008 in conjunction with the Twenty-Seventh International Conference on Conceptual Modeling (ER 2008). On behalf of the programme committee we commend these papers to you and hope you find them useful.

The primary objective of the workshop is to share research experiences in conceptual modelling for applications in life sciences and to identify new issues and directions for future research in relevant areas, including bioinformatics, health informatics, medical and veterinary informatics. The workshop invited original papers exploring the usage of conceptual modelling ideas and techniques for developing and improving life sciences databases and information systems. Following the call for papers which yielded 18 submissions, there was a rigorous refereeing process that saw each paper refereed by three international experts. The six papers judged best by the programme committee were accepted and are included in this volume.

We are grateful to Professor Victor Maojo from the Universidad Politécnica de Madrid who kindly agreed to present the CMLSA keynote address on Ontologies in Practice: From Biomedical Informatics to Nanomedicine.

We wish to thank all authors who submitted papers and all workshop participants for the fruitful discussions. We also like to thank the members of the programme committee for their timely expertise in carefully reviewing the submissions. Finally, we wish to express our appreciation to the local organisers at the Universitat Politècnica de Catalunya and the Universitat Oberta de Catalunya for the wonderful days in Barcelona. 